\title{
A Numerical Analysis of Riveted Lap Joint Containing Multiple-site Damage
}

\author{
Dazhao $Y U$ \\ Naval Aeronautical and Astronautical University, Yantai, Shandong, 264001, P. R. China
}

Received: 7 Oct. 2012, Revised: 26 Jan. 2013, Accepted: 28 Jan. 2013

Published online: 1 Jun. 2013

\begin{abstract}
The evaluation of stress intensity factors (SIFs) for cracks in mechanically fastened joints is one of the central issues in a damage tolerance analysis. Accurate stress intensity solutions may be difficult to determine due to geometric complexity along with variations in fastener load transfer and fastener interference and material thickness because of corrosion. Detailed finite element models that include specific aspects of fastener for lap joints analysis, but such representations are often impractical for large lap joints involving many fasteners. In order to reduce the number of degrees of freedom in a particular model, a methodology was implemented that efficiently depicts mechanical fasteners in lap joints using finite elements. Uncorroded and corroded lap joints with three crack scenarios were studied by the computationally efficient model. The effects of pillowing corrosion and fastener interference were also included in the model. The results show that the effect of MSD and thinning of the material is to increase substantially stress intensity factors (SIFs) values compare to that of a single crack without corrosion. For a given cyclic stress range, SIF decreases with increasing rivet interference level. This is particularly true for shorter crack lengths. SIF values for the longer crack are not sensitive to the length of shorter crack on the opposite side of the rivet hole. The effects of corrosion pillowing cause SIF values on the faying surface to be larger than those on the opposing surface, and the crack front distorts from the straight front. Moreover, stresses on the faying surface may exceed the yield strength of the material.
\end{abstract}

Keywords: lap joint, stress intensity factor, multiple site damage, fastener interference

\section{Introduction}

Multiple site damage (MSD) cracks have been of great concern since the Aloha accident in April 1988 [1-7]. The coalescence of MSD cracks may lead to sudden fracture and adversely affect the damage tolerance of structures. This is because the anticipated failure load will be significantly underestimated if consideration is taken solely of the fracture resistance of a single crack. MSD is widespread fatigue damage with the unusual feature that small fatigue cracks appear at multiple rivet locations along the lap joint. One central issue in quantitatively evaluate the effect of MSD cracks on the structure integrity is evaluating stress intensity factors (SIFs) for MSD cracks at rivet holes in mechanically riveted joints.

Accurate stress intensity solutions may be difficult to determine due to geometric complexity along with variations in rivet load transfer and rivet interference and material thickness because of corrosion, especially the interaction of MSD cracks. Detailed finite element models of the structure and associated rivets can be used to estimate the state of stress in lap joint. However, it is often impractical to explicitly model specific details of rivets geometry for a significant number of rivets. In order to reduce the number of degrees of freedom (DOFs) in a particular model, individual rivets connecting adjacent layered structures may be idealized using simple spring elements [3]. Of course, the adopted spring force-displacement relationship has a substantial effect on the load transfer in a given joint as well as on the SIF solutions for any cracks in the joint [8]. Cope and Lacy verified this approach by considering different combinations of explicitly modeled fasteners and spring-rivet fasteners in single shear lap joint models with three rows of fasteners (cf. Figure.1.1 for three such models).

Based on previous investigation, this study investigates the accuracy of the efficient modeling methods to determine SIFs for riveted lap joints with MSD. The influence of rivet interference and corrosion damage on SIFs solutions was also investigated.

\footnotetext{
*Corresponding author e-mail: ytyudazhao@yahoo.com.cn
} 


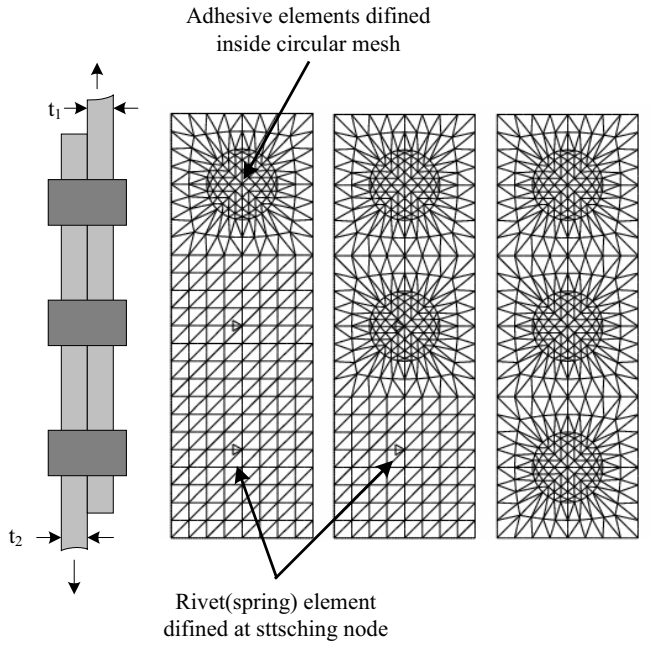

Fig. 1 Lap Joint Models with Various Rivet Model Combinations

\section{Analysis Approach and Tool}

Carleton University and National Research Council of Canada (NRC) have completed several fatigue tests on uncorroded and corroded lap joint specimens. Figure.2.1 shows the geometry and material [10]. Test specimens have been specially designed to simulate aircraft conditions, in particular the development of MSD and the MSD failure model. The key feature of the concept is the use of bonded side straps to simulate the load transfer from cracked areas to the surrounding structure that occurs on aircraft. This allows MSD cracks to develop and linkup premature failure of the specimen. The specimens were constructed of two 2024-T3 clad aluminum sheets with a thickness of $1 \mathrm{~mm}$ and a width $25.4 \mathrm{~mm}$. Sheets were connected to a doubler with a thickness of $4 \mathrm{~mm}$. The edges of the spliced sheets were reinforced by straps that were bonded together.

In order to verify that if Cope's method suite for lap joint with MSD, a stress analysis was performed to determine crack tip SIF values of MSD lap joint specimens (cf., Figure.2.1).

In analyzing the crack growth test data, it was observed that $95 \%$ or more of the joint life was controlled by the lead crack behavior. $50 \%$ of the occurrences were a single crack (Scenario 1), 10\% were double cracks with equal length (Scenario 2), and the remaining $40 \%$ were double cracks with different lengths on each side of a rivet (Scenario 3). The thickness loss estimated in the corroded specimens was about $2 \%$. Analysis was only preformed for the lead crack using three different scenarios, as observed in the test data. The first scenario consisted of a single, radial crack emanating from a single rivet near the center of the specimen. The second scenario consisted of double cracks of equal length emanating from a single rivet near the center of the specimen. The third scenario consisted of double cracks of differing lengths emanating from a single rivet near the center the specimen.

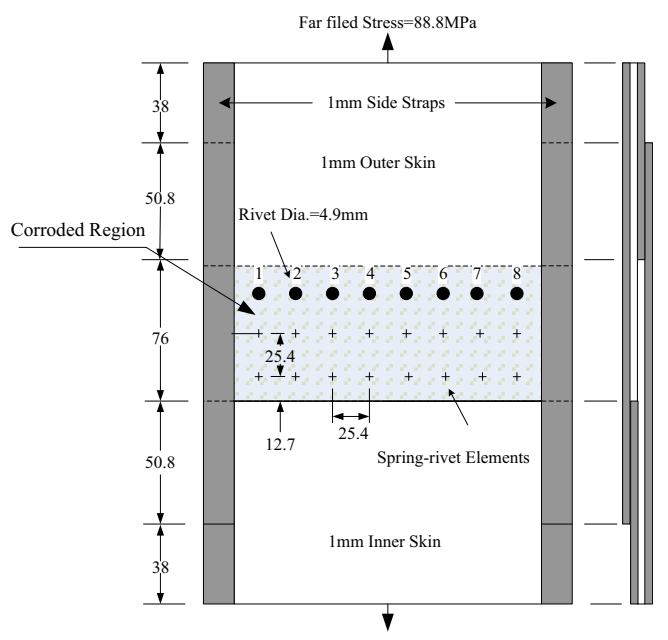

Fig. 2 NRC Lap Joint Specimen Configuration (unit: mm)

The special purpose code FRANC2D/L[11] was used in this study to determine crack tip SIF values of MSD lap joint specimens. FRANC2D/L is a highly interactive finite element program well suited to performing small deformation analyses of two-dimensional layered structures. The mode allows for efficient SIF determination and incorporates an adaptive remeshing algorithm that facilitates mixed-mode crack propagation and incorporates an adaptive remeshing algorithm that facilitates mix-mode crack propagation. The layered capability allows the user to model mechanically fastened and/or adhesively bonded structures, such as planar lap joints and bonded repairs. Individual structural components such as skins, stiffeners, and doublers may be modeled as separate layers in FRANC2D /L, each with its own associated finite element mesh. Overlapping layers may be defined with coincident mid-planes or with an appropriate mid-plane offset to account for joint eccentricity. FRANC2D/L uses a linear bending algorithm to automatically calculate the bending stresses/strains and out-of-plane displacements in addition to the standard in-plane stress, strain, and distributions.

\section{FRANC2D/L Model}

Cope and Lacy modelled single shear lap joints with three rows of mechanical fasteners using different combinations of explicit and spring element 
representations of fasteners in order to determine the appropriate level of model refinement necessary foe accurate SIF solutions for cracks emanating from fasteners holes. The results suggest that combinations of explicit and spring element representations of fasteners may be used to develop computationally efficient lap joint models while retaining the essential features of the fastener displacement and load transfer within the joint. In order to reduce the number of DOFs in lap joint model, individual rivets connecting adjacent layered structures were used simple spring element in this study.

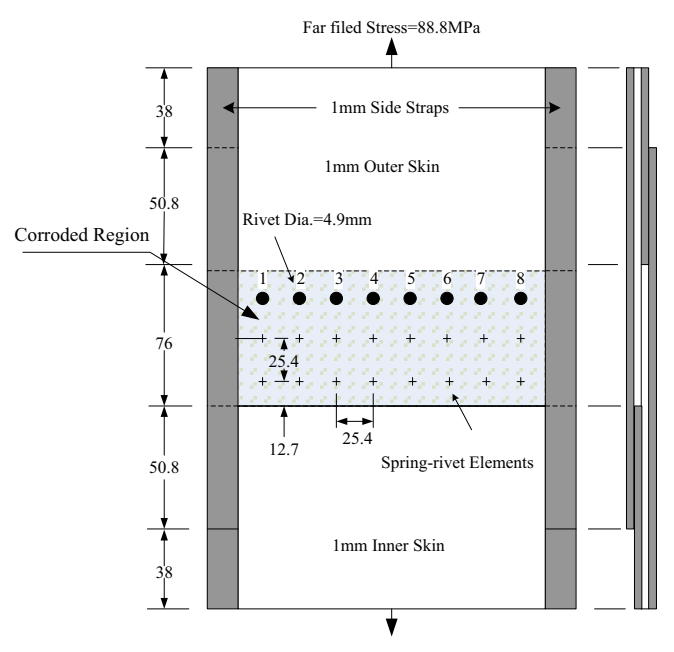

Fig. 3 Mesh of Outer Layer used for FRANC2D/L SIF Calculations

The FRANC2D/L model configuration (cf., Figure.3.1) was based on the geometry, material, and loading of the experimental specimens. The model consisted of six-noded triangular plate elements. The inner and outer skins, modelled as discrete layers, were attached only at the rivet locations and along the side straps.

Material properties for the adhesive elements and for the spring-rivet elements were selected in order to provide reasonable estimates of the relative rivet displacements and load transfer between the layers. When modelling mechanically riveted lap shear lap joints using a combination of explicit and simplified spring representations of rivets, use of equations(1)-(4) to define the rivet properties ensures that the simplified spring elements will provide approximately the same load transfer and relative displacement between sheets as explicitly modelled rivets.

$$
E_{F}=\frac{8 E h}{\pi d}\left[(1+v)+\frac{8}{3}\left(\frac{h}{d}\right)^{2}\right]\left[B+C\left(\frac{d}{t_{1}}+\frac{d}{t_{2}}\right)\right]^{-1}
$$

$$
\begin{gathered}
G_{F}=\frac{E_{F}}{2(1+v)} \\
k_{s}=\frac{\pi d^{2} G_{F}}{4 h} \\
k_{b}=\frac{3 \pi d^{4} E_{F}}{64 h^{3}}
\end{gathered}
$$

where

$E=$ sheet material elasticity modulus;

$h=$ joint eccentricity; $v=$ Poisson's ratio;

$t_{1}=$ thickness of sheet in Layer1;

$t_{2}=$ thickness of sheet in Layer2;

$k_{s}=$ shear stiffness; $k_{b}=$ bending stiffness.

$B=5.0, C=0.8$, for aluminium rivets.

Rivets were modeled using two methods. In the first method, the rivets were modeled as circular, elastic plugs in both layers, and the plugs are attached to the surrounding structure using gap elements. The second method of modeling rivets were modeled by one-dimensional elastic-plastic rivet elements that connect shell finite element nodes in the upper and lower skins. Each rivet element has six degrees of freedom, corresponding to extension, shearing, bending and twisting of the rivet. The stiffness of each degree of freedom was defined by prescribing a force-deflection curve. The axial, flexural, and tensional stiff nesses were computed by assuming that the rivet behaves like a simple elastic rod with a diameter of $4.9 \mathrm{~mm}$. The elastic shear stiffness of the rivet element was computed by the equations (3)-(4).

Contact interface elements were applied to the rivets in the upper row. Load eccentricity inherent to the single lap joint problem was introduced by offsetting the two skin layers. The effect of material thinning was modeled through a uniform thickness reduction over the corroded region.

In addition to the effects of MSD, material thinning due to corrosion damage was also studied. The effect of material thinning was modeled by a uniform reduction in thickness of the upper skin in corroded region. Three levels of corrosion were considered, no corrosion and corrosion equivalent to $2 \%$ and $5 \%$ of the material thickness.

The effect of the transverse pressure to model pillowing was to induce high local bending stresses in the skins around each rivet, thereby producing non-uniform stresses through the thickness of the skin.

In order to investigate the effect of rivet interference on the predicted load transfer and SIFs, rivet interference were also modeled by adjusting the stress-displacement curve in the interface elements to produce a residual circumferential compressive stress around the rivet hole.

Cracks were introduced into the outer skin in upper rivet row according to three different scenarios. 


\section{FRANC2D/L Analysis Results}

Results from the FRANC2D/L analysis of the test specimen revealed that a load distribution of $35 \%$ in the lead trailing rows and $30 \%$ in the center rivet row. The results matched strain gage data reported in the experimental study [8]. This suggests that large lap joint models may be constructed using both explicit and spring element representations of rivets without a serious loss of accuracy.

The K-solutions obtained from the FRANC2D/L analysis were recorded and converted to beta factors. The conversion equation is given below:

$$
\beta=\frac{K_{\max }-K_{r e s}}{\sigma_{r e f} \sqrt{\pi c}}
$$

Where $\mathrm{K}$ is the stress intensity factor, $\sigma_{r e f}$ is the reference stress based on experimental load level [8], and $\mathrm{c}$ is the crack length.

To evaluate the effect of MSD cracks, the cracks of three different scenarios were introduced into the outer skin in upper rivet row.

Figure.4.1 compares the beta results for the three crack configurations corresponding to Scenarios 1 through 3. The plot shows a significant increase in beta for the case of diametrically opposed cracks compared to that of a single crack, as has been observed in test studies. With $\mathrm{c} / \mathrm{r}$ increasing, this phenomenon is more apparent. Results for Scenarios2 and 3 show very similar beta values, which indicate that Beta values for the longer crack in Scenario 3 are not sensitive to the length of shorter crack on the opposite side of the hole. Scenario 2 provides an upper bound to beta values. Since Scenario 2 and 3 yielded such close results, Scenario 3 was omitted from most of the subsequent analysis.

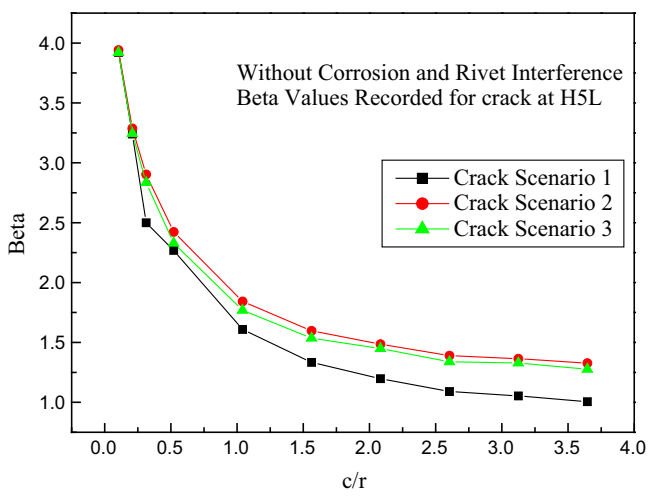

Fig. 4 Beta Predictions for Different Crack Scenarios

It has been shown that rivet interference significantly impacts crack growth by reducing the initial crack growth rates [3, 9-10]. In order to investigate the effect of rivet interference on SIFs, four different levels of interference $(0.00 \%, 0.25 \%, 0.5 \%, 0.78 \%)$ were studied.

The betas were calculated to assess the effect of various interferences on beta, as shown in Figure.4.2. As expected, for a given $\mathrm{c} / \mathrm{r}$, beta decreases with increasing rivet interference level. This is particular true for shorter crack length.

The effect of corrosion was modeled through uniform thinning of the material in the corroded region. In this case, one would expect the average stresses in the corroded region, and therefore the beta values, to increase by a factor of approximately $\mathrm{t} / \mathrm{t}_{\text {corr }}$, where $\mathrm{t}$ is the original thickness, and $\mathrm{t}_{\text {corr }}$ is the corroded panel thickness. The FRANC2D/L prediction yields the expected result, as the beta values for the $2 \%$ and $5 \%$ corrosion case are approximately 1.02 and 1.05 times higher than for the case with no corrosion respectively (cf., Figure .4.3). The results were consistently obtained for all analysis cases which had zero rivet interference.

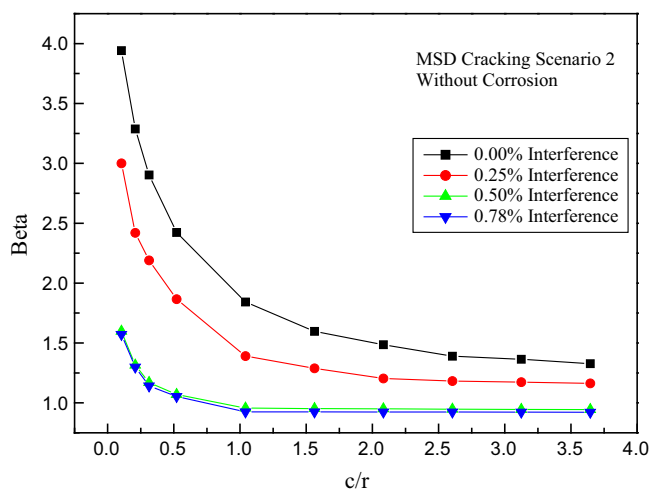

Fig. 5 Beta Predictions for Different Levels of Rivet Interference

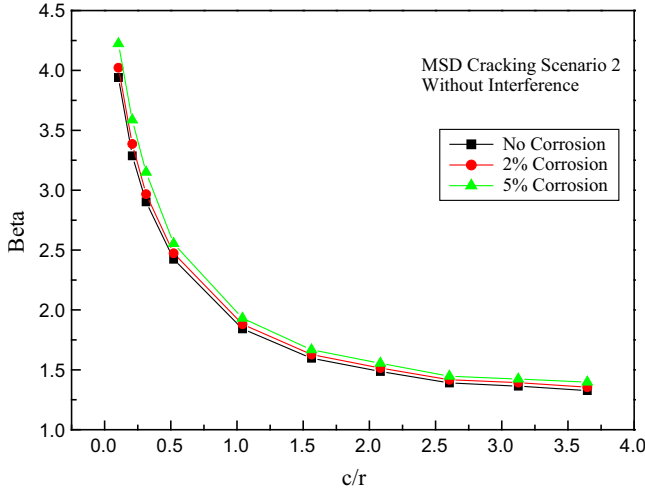

Fig. 6 Beta Predictions for Different Corrosion Level

The results also show that the effects of corrosion pillowing cause SIF values on the faying surface to be larger than those on the opposing surface, and the crack front distorts from the straight front. Moreover, stresses 
on the faying surface may exceed the yield strength of the material, producing plastic deformation.

It should be recognized that linear two-dimensional finite element analysis of riveted lap joints may be ill suited for the case where the out-of-plane displacements become large relative to the sheet thickness. A full three-dimensional analysis may be necessary in order to appropriately account for the influence of bending effects due to joint eccentricity on the load transfer and calculated peak SIFs. This key issue, as well as development of idealized rivet reorientations for use three-dimensional finite element models, will be the focus of further investigations.

The last scenario investigated combined all the previously discussed effects: MSD crack, and corrosion. Each of these effects was modeled using the methods previously discussed.

Figure.4.3 compares beta for MSD, corrosion, and combined scenario. It is evident that MSD and corrosion damage especial combined scenario significantly increase SIF values compare to that of a single crack.

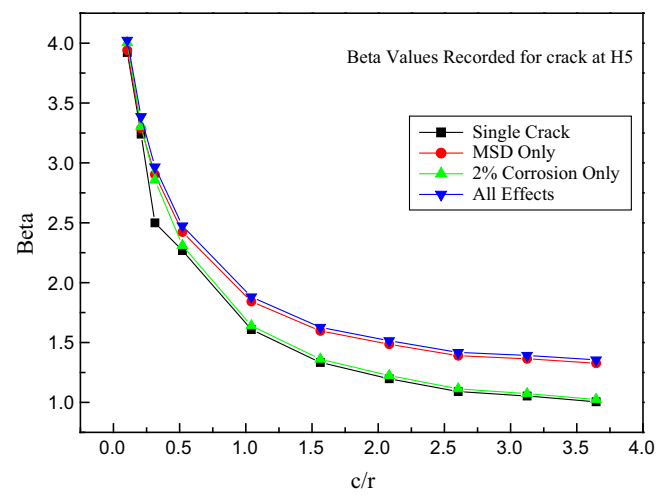

Fig. 7 Combined Scenario: MSD and Corrosion

\section{Conclusion}

A methodology for efficiently depicting rivets in lap joints using finite elements was implemented in order to facilitate numerical analysis of lap joint with MSD. FRANC2D/L model of MSD lap joint was modeled using a combination of explicitly modeled rivets and spring element representations of rivets. SIF solutions were obtained for three scenarios MSD. These results suggest:

1. Combinations of explicit and spring element representations of rivets can be used to develop computationally efficient lap joint models while retaining the essential features of the deformation and load transfer within the joint. Explicit representations of fasteners may be incorporated wherever an accurate depiction of the local stress field is required, such as in the vicinity of cracks emanating from rivet holes.

2. The effect of MSD and corrosion is to increase substantially beta values compare to that of a single crack without corrosion.

3. For a given cyclic stress range, the beta decreases with increase rivet interference level. This is particularly true for shorter crack lengths. When the rivet interference reaches special value, its effect on SIF will not greatly change with interference increasing.

4. Beta values for the longer crack are not sensitive to the length of shorter crack on the opposite side of the hole.

5 . The effects of corrosion pillowing cause beta values on the faying surface to be larger than those on the opposing surface, and the crack front distorts from the straight front. Moreover, stresses on the faying surface exceed the yield strength of the material. In this paper, we analyzed WDM optical communication network structure, discussed the add-drop system and proposed a new routing selection method. Routing technology plays an important role in a optical communication network. We discussed the routing design and switching technology for WDM network in optical networks. Routing is the process of selecting paths in a network along which to send network traffic. A good routing selection method can make the network do better performance.

\section{Acknowledgements}

The present work is part of the contribution of National Natural Science Foundation of China(No. 50675221). Thanks for the help.

\section{References}

[1] D.A. Cope and T. E. Lacy, Stress intensity determination in lap joints with mechanical fasteners, 41st Structures, Structural Dynamics, and Materials Conference on Atlanta. 1, 3-6 (2000).

[2] N.C. Bellinger and J.P. Komoroski, Corrosion pillowing stresses in fuselage lap joints. AIAA Journal. 35, 317320(1997).

[3] D.Z. Yu, Study on mechanical properties of bolted joints based on experiment and three-dimensional finite element, Journal of Mechanical Strength.,33, 855-861(2011).

[4] Y. Shang, Optimal attack strategies in a dynamic botnet defense model, Applied Mathematics and Computation.6, 29-33(2012).

[5] M.T. Alquran, Solitons and periodic solutions to nonlinear partial differential equations by the sine-cosine method, Applied Mathematics and Computation.6, 85-88(2012).

[6] S. Furuichi and H. Hino, Mathimatical analyses of 2010 FIFA word cup, Applied Mathematics and Computation.5, 205-219(2011). 
[7] N. Chen, Numerical simulation and experimental researches on the LED reliability under temperature loading, Applied Mathematics and Computation.6, 775-779(2012).

[8] D.S. Dawicke, E.P. Phillips and D.V. Swensen, Crack Growth from Loaded Rivet Holes, International Symposium on the Structural Integrity of Aging Airplanes. 44-51 (1992).

[9] M. James and D. Swenson, FRANC2D/L: A Crack Propagation Simulator for Plane Layered Structures. Version 1.5 User's Guide, Cornell University. 45-60(2002)

[10] J.C. Newman, C.E, Jharris and M.A. James, FatigueLife prediction of riveted lap splice joints using smallcrack theory. 19th International Committee on Aeronautical Fatigue Symposium on Edinburgh, Scotland, 18-20(1997)

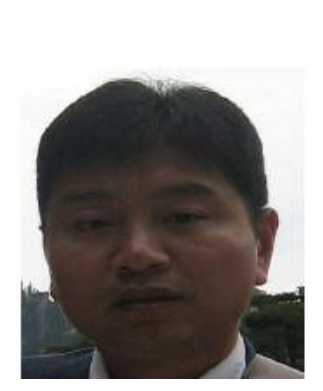

\section{DazhaoYu}

received the MS degree in Mechanics science from Naval Aeronautical and Astronautical University in 2003, and the $\mathrm{PhD}$ degree in Mechanics science from Naval Aeronautical and Astronautical University in 2008. He is currently a professor in Naval Aeronautical and Astronautical University. His research interests are in the areas of the effects of MSD on fatigue life of aircraft. 